\title{
We Are All Romantics and That is Fine
}

\author{
KEVIN HIRTH \\ University of Colorado Denver
}

\section{A CONSTRUCTED CONTEXT...}

The image of our present is a stone-cold, inanimate, non-existent, fizzing thing. It is perfectly suited to a life of duplicity. Today, the image appears far more comfortable flitting across a screen than being fixed in a frame and hung from a nail in a museum. Better to live fast and die young than suffer the ignominy of achieving some fixity and then watching the world grow and move on without you. At the heart of this identity, the image of our present is increasingly surrendered to the subjectivity of the observer. This act of distortion opens up alternate readings of the individual image based on the ambiguity of the intent of its author.

Over the past decade the image has evolved remarkably from its traditional role as a record of the past into serving a more viscous and atemporal one. Images are shared and distributed virtually with little governing their interpretation. Images have shifted from "speaking a thousand words" and providing inalienable truth of a place and time to becoming promiscuous in both representation of that time and of the notion of a fixed reality. The image has become elusive, concealing some deeper truth to suspend any immediate understanding. Passed from individuals into the collective consciousness via complex webs of interaction, images often elude authorship and are unmoored from the recorded moment that they may represent. Often manipulated, often artificial, the twenty-first century image is a illusion.

Preceded in tradition by artists and architects across history, the nature of the architectural image as a resonant fantasy is indicative of a discipline reflective of the moment that contains it. By accepting permissible falsehoods and honestly projecting the fabricated nature of architectural imagery, the work of the architect in practice often demonstrates a dispensation of technical skill while cycling through acceptable architectural tropes and pushing into novel conceptual terrain. This cycling (and recycling) of common tricks familiarizes the subject while demanding increasingly diverse narrative forms. The image of architecture is always a collection of parts bringing about a whole. Whether it is a simple photograph of a new building on an existing site, or a collage of disparate objects placed together to create a compositional whole, the image of architecture is a composite of things synthetic and sometimes real or whole. This act of visual collection represents the construction of an artificial context, and the predominant body of work of the architect.
These collections, or illusions of reorientation take a number of forms that can be categorized with precedent into a conceptual framework of four parts. The first, a constructed illusion of realism, is pervasive in contemporary practice and waning as it is exhausted. The second, a manufactured context of structure, uses disparate and often seemingly unrelated relationships between objects to generate an impression of a conceptual whole. The third, an illusion of space, uses flatness to strip a clear registration of the third dimension from the contents of the image. The fourth, a generative camoflage of coincidence, casts objects into a singular whole by camouflaging them into one another.

\section{...OF REALISM}

The shift to the disposability and malleability of the image is central to the questions of our discipline today. Take a tour through the past five centuries of art and architecture to see how exhilaratingly violent the shift of the role of the image over the last decade has truly has been. Prior to the Renaissance, images were made to record or commemorate some fixed history, and often done so with strict rigidity and structure. Following this, through the lens of art historian and critic Dave Hickey, we find that there was a shift in temporality: For three centuries, illusionistic images aimed to slow life down - to make visible the fluid, violent, and often invisible constituents of temporal cultural experience. Then, in the nineteenth century, with the apotheosis of modernity, artists stopped slowing down life into images and began slowing down the images themselves. Thus in modern painting, our comfort level with illusion is always a matter of how exquisitely we delay the illusion's taking hold, since, for ideological fashion, we must always pay obeisance to the material present before we experience the imagined past. ${ }^{1}$

In the spirit of Hickey's observation, the image has moved inexorably from recording the past-real or imagined-to transmitting the present. The illusion generated is no longer one of transposed presence brought from the past but rather a presentation of current occupation. This blurring of present, past, and future into an image generates an effect of dislocation from reality to some unexpected meta-reality in which circumstances are idealized and contingencies are irrelevant. Images are made and circulated with the end-result of conveying a sense of constant production without conclusion. This effect is compounded by the pervasive use of photo editing software to distort and accentuate the properties of an image in order to affect the greatest response from a 


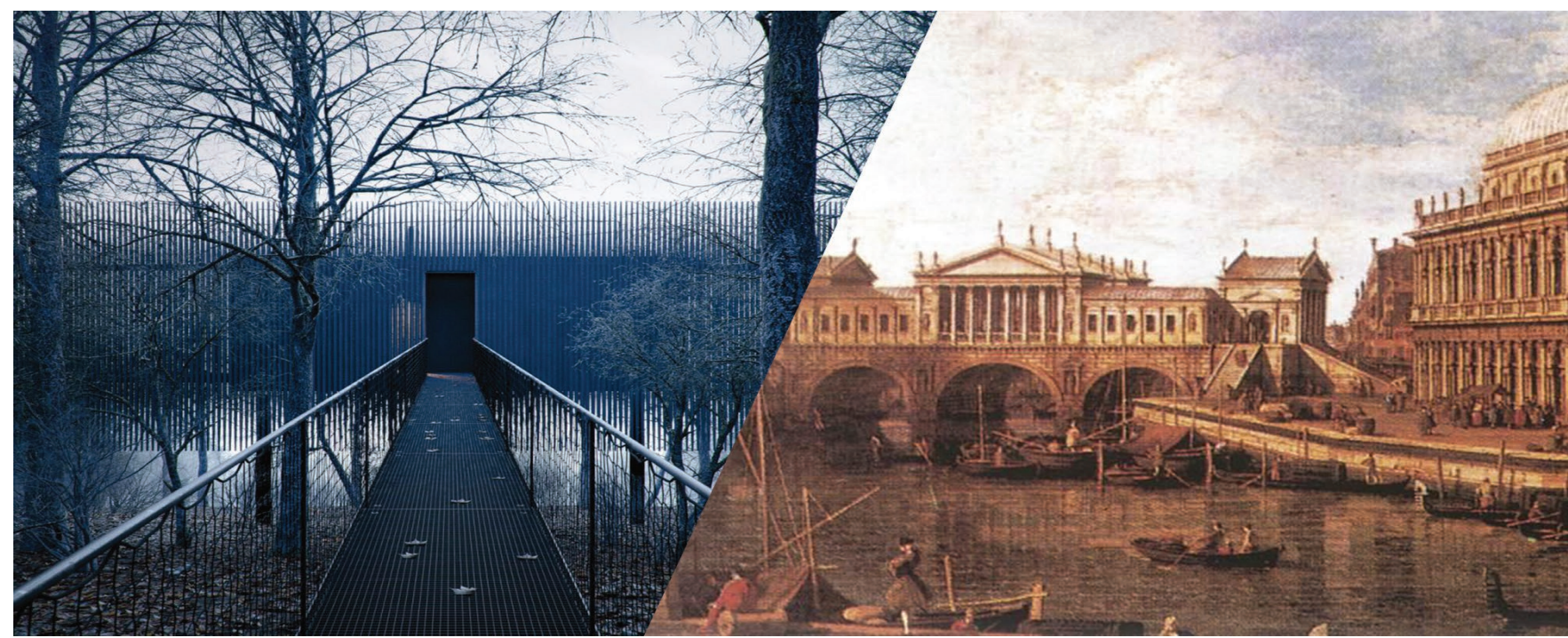

Figure 1. William O’Brien Jr / Canaletto Both forthright falsehoods.

captive audience. Images are created, edited, distributed, and circulated at an astounding rate, feeding a compulsive consumer culture of architectural imagery. By accepting that the image is no longer a bearer of intentional truth, the author and audience of a given image accept that they are viewing an alternate reality. Much like the audience of a magic show, we suspend disbelief and entertain a false reality in order to enjoy the experience.

This is readily apparent in a wide array of current works such as William O'Brien Jr's photorealistic renderings for "The Mask House," playful in their superficiality. The renderings, like many put forward in practice today, strive to achieve a clear illusion of reality. O'Brien puts forward work that bears the hallmarks of architectural photography while obviously portraying a conceptual project. This is, as such, a work of virtuosity. It is not put forward in a dishonest manner, attempting to hide it's abstraction. Rather, the work plays on the marginal expectation in our present that an image needs to be authentic or not. Look no further than your local real estate listings to find absurdly doctored photos of a real environment. These falsehoods are accepted. Because of this ambiguity, the illusion put forward by WOJR is clear. The value of the work is not in it's reality, but rather in the projection of a reality in which it could exist. By being self-declarative about the falsehood that the images put forward, the work is an illusion of reality in a fluid state.

An obvious parallel to the deceptive realism of images coming from current practice, the Enlightenment's Italian capriccio presented a similar soft break from objectivity. Capriccio techniques allowed the artist a license to subtly omit, edit, reconstitute, and idealize a scene either real or imagined. Ringing with an optimistic light and casting Venice as a postPalladian paradise, Canaletto applied a revisionist rhetoric within his work. Smoothing topography, altering facades, and pasting into scenes buildings designed but never built, Canaletto was using Photoshop nearly three centuries before it existed, all the while selling his paintings to a British audience hungry for his sun-soaked vistas of an idyllic Venice that they had never visited. 'After seeing Canletti's (sic) view ... There was neither the symmetry nor the richness of materials I expected,' wrote one disappointed tourist to Venice in $1774 .^{2}$ Canaletto's revisionism provided a release from realism, and did so without concern for authenticity.

A common practice of our time, editing and reconstituting a scene no longer poses an ethical quandary. Our present model of visual proliferation has desensitized us to the "Photoshop Fails" and Instagram filters that give our own reality a Cannelettan gauze.

\section{...OF STRUCTURE}

While Canaletto smoothed the rougher lines of Venice into a more attractive whole, several centuries later Aldo Rossi found the technique of capriccio to be a pliable conceptual armature for a different sort of formal appropriation. Rossi employed an ascribed methodology of internally focused, psychoanalytical structuralism to his own work from his own individuated 'analogous' perspective. The buildings that he studied, referenced, and created ceased to carry importance based on their existence in the real world, but rather took on signification as a body of work collected in his own memory. He looked inward to his own personal context rather than to the particularities of the specific work he was carrying out.

This navel-gaze subjectivity presupposes that the individual author is beholden only to themselves and is building a cumulative image of themselves and their own work that will ultimately formulate a complete picture once all of 


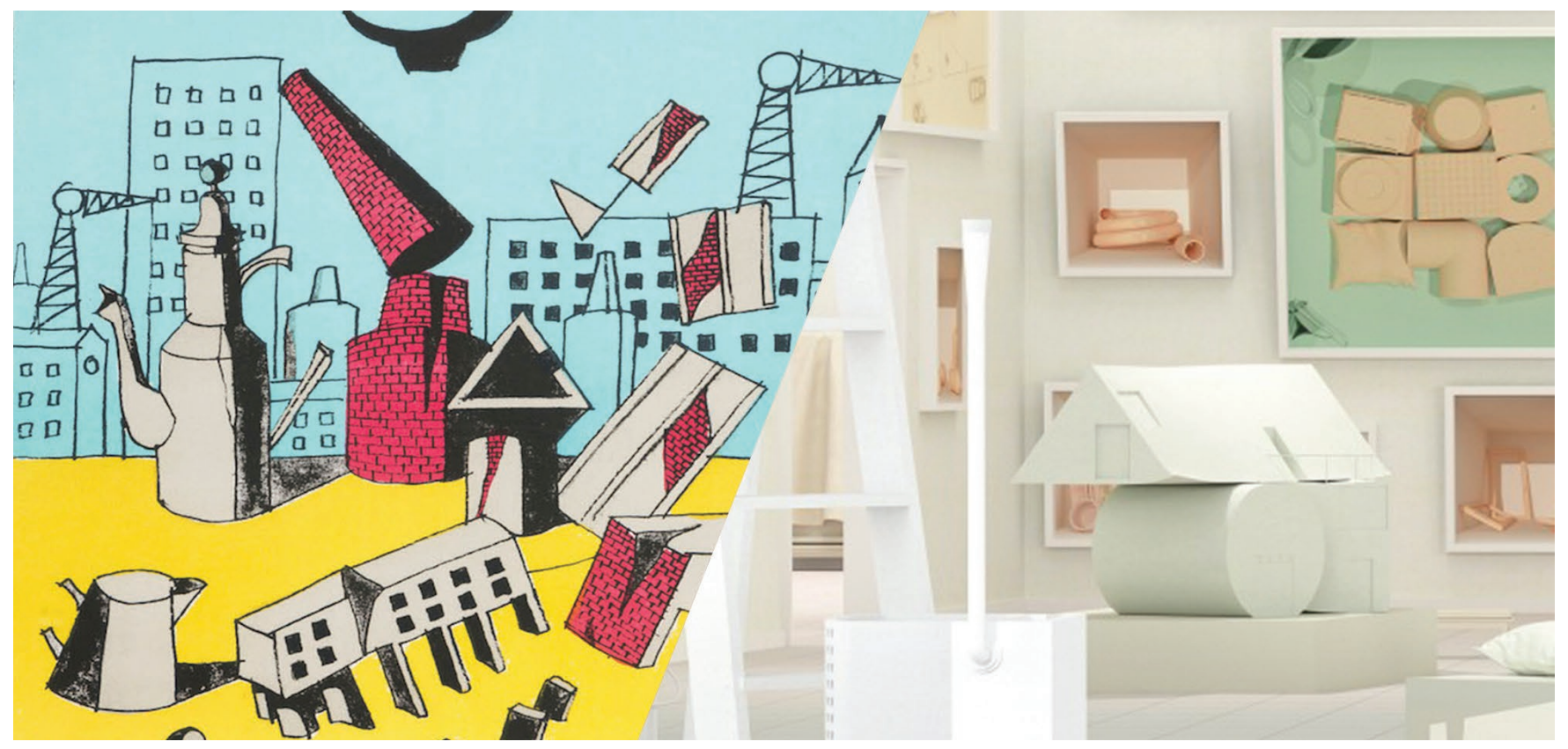

Figure 2. Aldo Rossi / NEMESTUDIO Both structures of association.

the individual pieces are ratcheted together into a single scene. This, a technique that Rossi employed in his noted 1976 entry to the Venice Biennale The Analogous City, has become unintentionally instructive in understanding the manner in which contemporary practices position themselves and their own work. As Pierluigi Nicolin once succinctly described: Both analogical and metaphorical meanings can, in this way, be ascribed to "The Analogous City" panel. That is to say, the work is open to a double, double reading. The panel is a montage composed of photo copies: fragments of urban plans (Como, the city of Vitruvius according to Gianbattista Caporai, in 1536), projects and drawings by Rossi himself (including a monumental coffee pot), Reichlin and Reinhart's proposal for the Castle of Bellinzona, hillside and lakeside views, a Piranesian quotation, and a finger-pointing figure by Tanzio da Varallo, a 17 th-century Lombardian painter. ${ }^{3}$

Rossi's method marks a departure from dispensing illusion using false realism in favor or generating a myth of coherence through the superimposition of incongruous parts into a collected whole. Neyran Turan and Mete Sonmez of NEMESTUDIO have elevated this practice into the present with a number of their recent projects. Most recently, their "Our Junk, Their Ruin" is a set of framed images portraying a virtual environment populated with models, tools, office supplies, and a space with walls covered in renderings of additional framed views. The coincidence of this imagery creates an impression that the work is a world collapsed in on itself. Real or imagined, these objects placed together create an interrelation of parts to the whole. A kerfed surface here is possibly drawing from a scalloped wedge there. The formal familiarity is instrumental in generating an imaginary order of completion. In Turan's own observation, "while depicting imaginary characteristics, one of the main features of the capriccio painting was its interest in versimilitude or slight (in)familiarity." This pursuit takes effective root in their contemporaneous work. The installation may not be a capriccio in fullness, but plays with the line of generating reality and falsehood simultaneously through the construction of an artificial context. ${ }^{4}$

Such associative practices have become rote in contemporary practice, as architects now surround themselves with the family tree of related and remote precedents in order to give signification to their work. The architecture of the present is constructed in a vacuum from unified coherent cultural association, and thus practitioners are returning to history and the arts to ground their work. This position, aptly demonstrated by Sam Jacob in his 2013 essay 'Faster, but Slower,' posits that contemporary architecture is severed from the modes, stylistic groupings, and generational iconoclasm of its conventional history. "Everything is deflated," he says, "collapsed into an indistinguishable pool."

Increasingly isolated, it is appropriate that practices spend so much time looking inwards rather than through colloquium. In searching for a coherent movement or disciplinary milieu, practices are increasingly reaching for the support of the evidence of things outside of the present moment. Like dumpster divers of the digital age, bargainbin online shoppers, contemporary practice demands that one seeks and finds the bespoke detritus that fell through the cracks. Such an approach is evident in the 2015-16 Chicago Architecture Biennial installation "House Is a House Is a House is a House is a House," by Johnston 


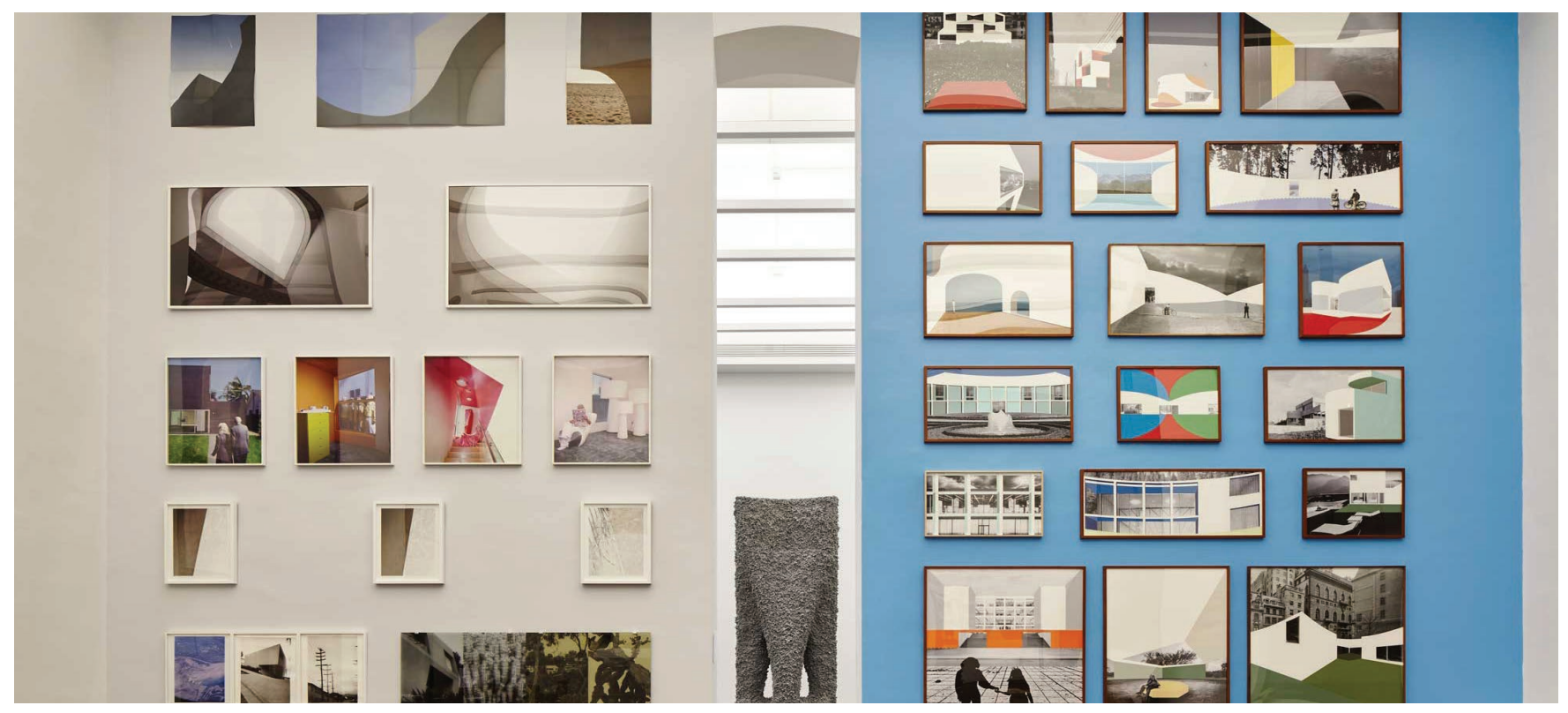

Figure 3. Johnston Marklee House Is a House Is a House is a House is a House.

Marklee for direct evidence of this search for signification. Johnston Marklee has established itself as an architectural firm engaged with contemporary art practices, drawing on artists, graphic designers, writers, and photographers to broaden the breadth of its research... In the early phases of a project, collage offers a way of drawing freely and quickly on diverse historical precedents, selecting key elements from existing architecture to establish a lineage with which the project will engage. ${ }^{6}$

Rossi and his collaborators were evidently foundational to this approach. The scope of the analogical project demanded that Rossi incorporate multiple associations, projects, and objects together. His work stitches together an analogous document that collects the buildings together with their influences alongside real and imagined urban contexts. This manner of collection is therefore entirely reliant upon the interrelated individual projects within Rossi's body of work to increase their signification. To borrow from Michael Meredith's 2015 essay "Toward the Body of Work": The ongoing cultivation of a body of work counters the instant amnesia of media by constructing collective relationships within and across time, between formats and buildings. Unlike a 'project,' the body of work focuses not on the dialectic between text and building, or the implementation of an ideology or an a priori idea, but rather on an ethos of production and the construction and curation of relationships between various buildings and other formats. ${ }^{7}$

That this practice finds increasing relevance today is indicative of a broader trend towards using the readily disposable image as a tool for contextualization and establishment of meaning regardless of motive.

\section{...OF SPACE AND TIME}

Again reflecting on Canaletto's continuing influence on successive architects, we turn to Britain, where Canaletto's work was purchased and circulated widely. Canaletto in fact relocated to London in 1746 in order to remain close to his largest market during an embargo between Britain and Venice. Canaletto and his work swept through the city to find the early seeds of Romanticism taking root. By 1833 Sir John Soane had officially handed his home over to Parliament to establish it as a museum. Upon his demise, the disciples of his legacy applied his collector's methodology to begin to elaborate their own visions of the hegemonic architecture of classical western culture. The result was a pervasive moment in which Soane's 'architecture as collection' and Canaletto's 'architecture as fantasy' co-mingled. Coopting the language of history and a use of Canaletto's capriccio techniques, a generation of architects began crafting their own illusions of reality that blur documented past and present along with reality and illusion.

Take C.R. Cockerell's "The Professor's Dream" (1848). Cockerell, who followed Soane both to the Royal Academy and as surveyor for the Royal Bank of England, picked up the mantel of the Grand Tour and returned to England brimming with collected architectural precedent. Cockerell's "Dream" draws into perspectival elevation an array of buildings both real and imagined, ascending and receding into the distance in increasing scale. These buildings exist together in a single sloping non-context, generating an atmosphere of congested activation by their asynchronous proximity to one another. Like an over-packed family group photo, distant cousins stand on their toes to get into the frame. Everything in the image is washed with a sepia tone, obliterating materiality and allowing the parts to gather into a coherent whole. 


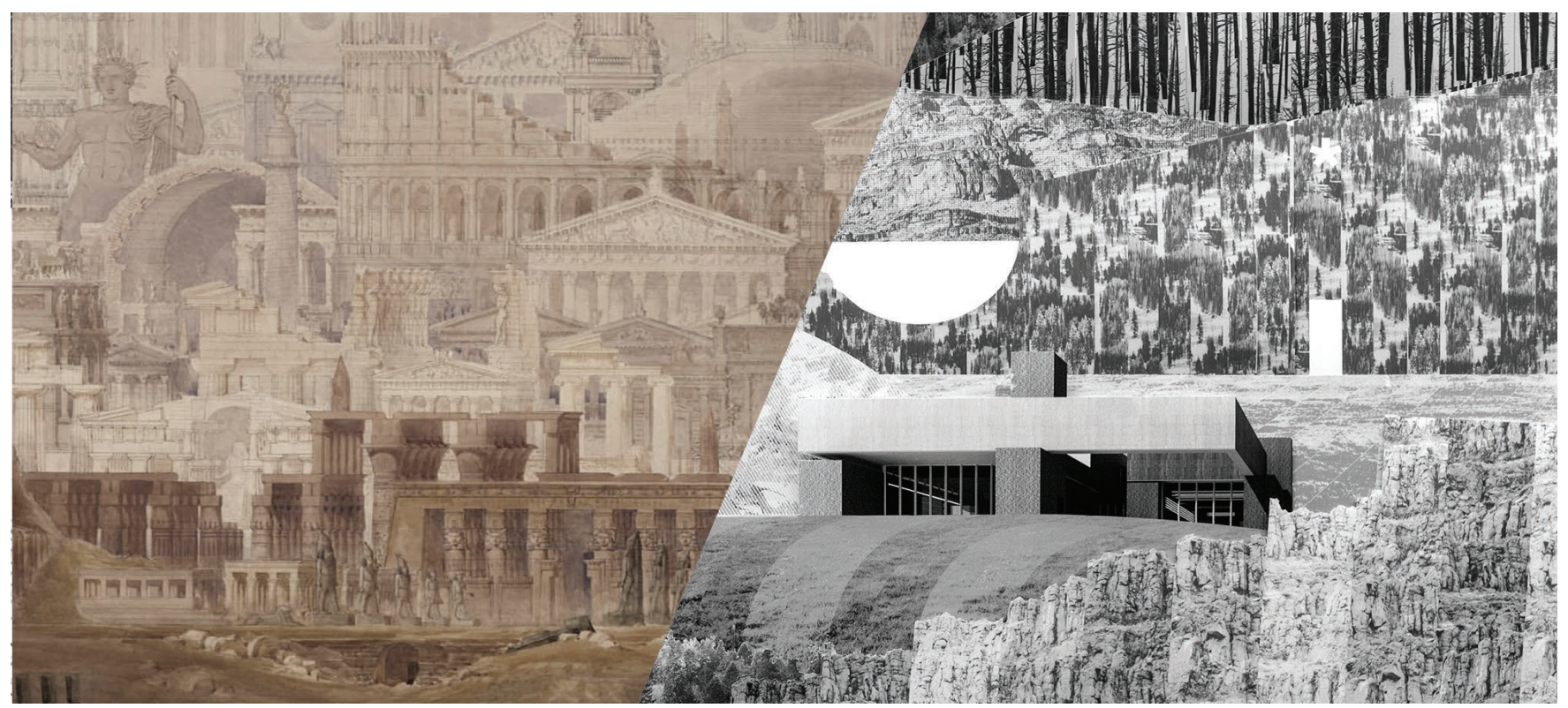

Figure 4. C.R. Cockerell / Kevin Hirth Both flattened adjacencies.

The effect is ultimately captivating more for its possibilities of adjacency and contradiction than as a visual catalogue of architecture. Cockerell has created an urban scene that foretells the sort of competitive monumental urbanity that the twenty-first century confronts as a normative condition, with layers of overlapping monumental buildings creating vertiginous negative space in between. The rendering of the individual buildings as a field of self-similar objects likewise introduces Cockerell as the editing author of all that is seen as his own. Because he has drawn them as a single overlapping composition, the observer easily reads the entire composition as a uniform monolithic construct. The result is an analogous image that approaches absurdity when divorced from it's indexicality. As Cockerell's biographer, David Watkin once wrote: This strange romance, these pyramids, porticoes, and domes, is surely the idea, years before its time, of Banister Fletcher's celebrated History of Architecture on the Comparative Method. One can only envy Cockerell for having lived before the invention of stylistic labels by architectural historians. Thus, unhampered by considerations of whether a particular building was 'Baroque' or 'Neo-Classical' he could dart from one period, place or person to another. ${ }^{8}$

A similar approach can be seen in my office's 2017 installation for the Architectural League of New York's League Prize. As is a typical practice for the annual exhibition supporting this award, my office sought to represent multiple previously completed projects in a gallery format. We sought to translate the standard practice of displaying projects on the wall adjacent to one another by coinciding all of the projects into a single unfolding and overlapping scene. The projects are cut and pasted into a collaged representation of the Colorado context in which they are fictionally situated but abstracted into something reflective of more primary elemental geometry. As a body of work viewed in total, each project supports the next to create a greater whole. Sparsely scattered among these projects are a series of blank figures, placed to imply anticipated future work. Unambiguously typological in nature, the projects use uncomplicated primary geometry in unexpected configurations to exist in concert with the grandiose context of Colorado. The work is cast as a set of characters arranged together to create a provocative scene. The models, made of the same paper as the collage, appear to be torn from the wall and draped over a table in front of it. Presented without scale and in duplicate, this work pushes the image into new territory as a vehicle for illusion by restating the Cockerell's approach in a different manner.

\section{OF COINCIDENCE}

With Cockerell's sweeping monumentality in mind, now take the painting "Various Designs for Public and Private Buildings" 1780-1815 by Joseph Michael Gandy. A collaborator and mentee of Soane, Gandy assembled his own representation of the analogous post-present architectural image. Gandy's shows a collection of architectural models and paintings stacked atop one another in a dark room. Lit from a brilliant circular lamp, the models appear to exist both at their given scale and as a representation of some alternate urban reality. This collection of built and unbuilt work, cast in this light, cracks open a critical question of the nature of the existence of these projects. Gandy sees the models and drawings of the buildings that he is painting as primary in themselves. He views this painting of the models and drawings as a non-scalar, analogous arrangement in which all of these works exist in a contemporaneous physical plane. 


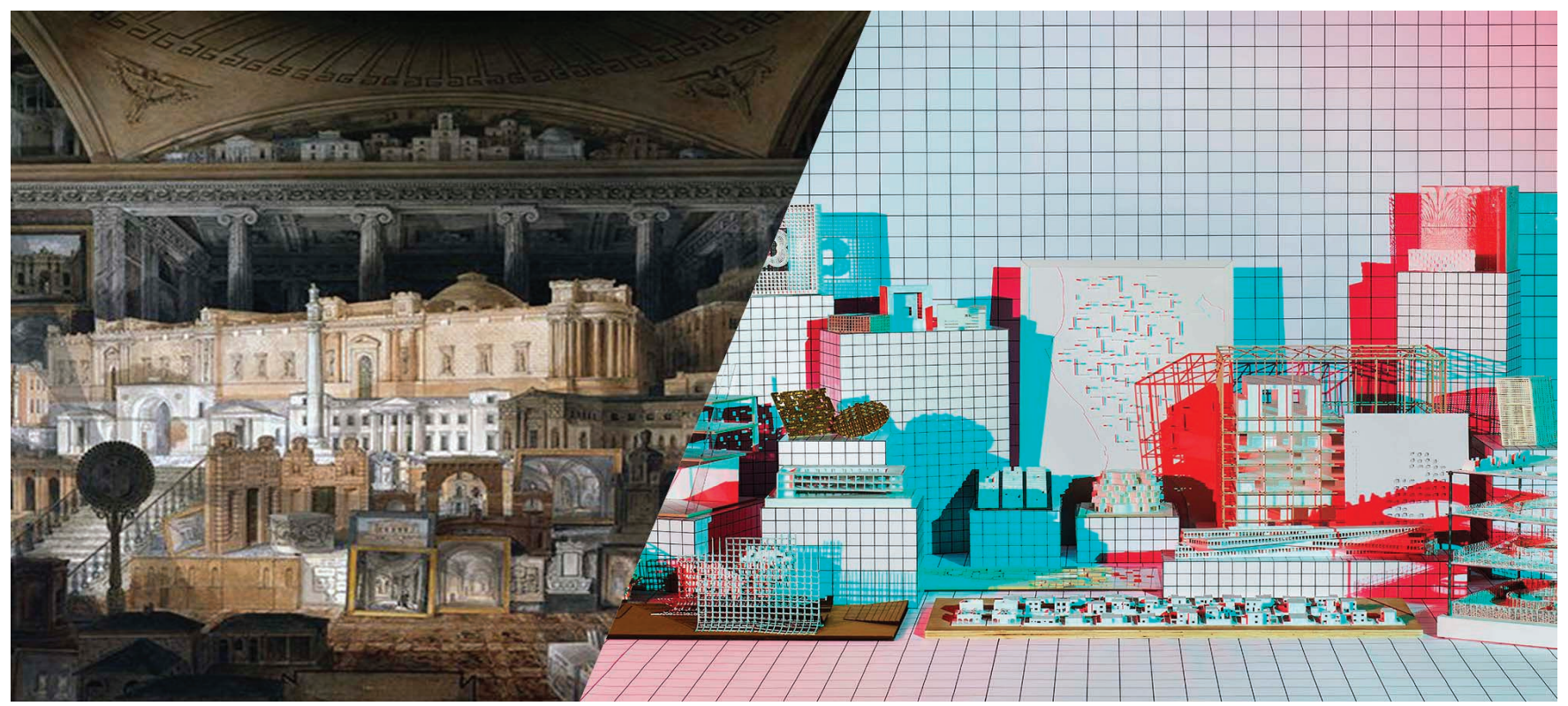

Figure 5. Joseph Michael Gandy / Adam Detour and Jennifer Bonner.

Parallel to this example, consider Adam DeTour's photographs for Jennifer Bonner's 2016 catalogue of Harvard GSD student work, Still Life. DeTour and Bonner "borrowed freely from 16th-century oil paintings as well as food photography-a contemporary cultural obsession. [They] referenced advertisements in high-fashion magazines, Caravaggio's Basket of Fruit (1565), Giorgio Morandi's obsessive tracings, and Umberto Eco's The Infinity of Lists (2009). Most Radically [they] experimented with color gels, partly in homage to the artist Barbara Kasten." ${ }^{\prime \prime}$ Through Bonner and DeTour's exhaustive work to recontextualize the student models in these photographs, we find the work in a temporary analogous reality. The images themselves have taken on their own significance, obliterating the intentions and contexts of the individual works contained within them to generate a new and optimistic context in colloquium.

The result is an expansion on Gandy's practices. Using abstraction, the scenes cast individual projects together in a literal light that binds them. They are represented as a singularity by obliterating any textural or tonal differences between them. They are likewise cast into an ambiguous scale by placing them into juxtapositional adjacency. The result is a propositional urbanity that could either be representational or merely happenstance.

\section{A COMPLETE PICTURE}

Architecture is inherently a pursuit of projection. In practice, the architect generates drawings that direct the creation of something from nothing. That architects would play openly with the image as a vehicle for the imaginary is not alien. The juxtapositions laid out in this paper point to a position in discursive practice that the past is a part of dealing signification to the present. These are all drawings that collect various parts into a fictive and contextual whole. The image conceptualized as a collection is demonstrated here in various roles, all dealing with relating the imaginary to reality. The common thread that ties these images is the disposition of something unreal as a meaningful gesture of architecture. These images are the production of a series of fully composite environments intended to generate a complete picture of the context containing them. The fantasy that is created through their unreality is a fundament of the architectural imagining. Why should we care whether something is real and authentic or if it is imaginary? The instantiation of something illusory is the core intent of architecture. As makers of images, architects are uniquely positioned to generate reality and authenticity whether it makes a mark in the concrete and real world or not. This tenuous position is what embodies the value of the works shown here as a map to an architecture of self-aware constructed contexts and a discipline in step with the fuzzy fluidity of the images of the present.

\section{ENDNOTES}

1 Dave Hickey, "This Mortal Magic," in Air Guitar: Essays on Art and Democracy (Los Angeles: Art Issues Press, 1997), 185-186

2 Rachel Spence, "Canaletto: Visions of a Venice Even Better than the Real Thing," Financial Times, June 6, 2017. https://www.ft.com/content/ ec080af4-3f07-11e7-82b6-896b95f30f58.

3 Pierluigi Nicolin, "Tafuri and 'The Analogous City,' ANY: Architecture New York 25/26 (2000): 16-20

4 Neyran Turan, “Can Images Implode?" Room One Thousand 5 (2017): 188-198.

5 Sam Jacob, "Faster, But Slower," Log 29 (2013): 145-152.

6 Sharon Johnston and Mark Lee, "House Is a House Is a House is a House is House," in Chicago Architecture Biennial Guidebook, eds., Joseph Grima and Sarah Herda (Chicago: Chicago Architecture Biennial, 2015): 44.

7 Michael Meredith, "Toward the Body of Work," Log 35 (2015): 11-14.

8 David Watkin, The Life and Work of C.R. Cockerell (London: Zwemmer Books, 1974), 132

9 Jennifer Bonner, Platform GSD: Still Life (New York: ACTAR, Harvard Graduate School of Design, 2016), 8-9. 